\title{
Interference Effects of Multiple Excited States in the Resonance Raman Spectroscopy of $\mathrm{CpCoCOD}^{\dagger}$
}

\author{
Susan E. Bailey, Judson S. Cohan, and Jeffrey I. Zink* \\ Department of Chemistry and Biochemistry, University of California, Los Angeles, \\ Los Angeles, California 90095
}

Received: February 4, 2000

\begin{abstract}
The resonance Raman excitation profiles of cyclopentadienylcobalt cyclooctadiene, CpCoCOD, contain pronounced decreases in intensity in the region of the lowest energy ligand field absorption band. This deenhancement is caused by interference between the ligand field state and nearby metal to ligand chargetransfer states. The changes in the Raman intensities are quantitatively calculated. The relevant electronic excited states are assigned by using single-crystal absorption spectroscopy, and the vibrational modes are assigned by deuteration studies. Distortions along the metal-ligand vibrational normal coordinates in the interfering states are calculated. The origin of the deenhancement, the relative signs of the bond length changes, and the relationship of the bond length changes to the molecular orbitals involved in the transitions are discussed.
\end{abstract}

\section{Introduction}

Resonance Raman excitation profiles, plots of the intensities of bands as a function of the wavenumber of the excitation laser, usually have maxima at the same wavenumber as that of the absorption band with which the excitation source is in resonance. The relative intensities of the profiles are related to the bond length and angle changes in the normal coordinate giving rise to the band that is measured. ${ }^{1-6}$ However, under specific conditions, an excited electronic state nearby in energy to the state of interest can produce significant interference effects that lead to a minimum in the profile at the wavenumber near that of the absorption band maximum. This loss of intensity at an excitation energy where a maximum is expected is called "resonance deenhancement". ${ }^{7-12}$ Theoretical treatments that use the Kramer-Heisenberg-Dirac (sum-over-states) method ${ }^{11}$ or the time-dependent theory of resonance Raman spectroscopy ${ }^{12,13}$ have been published.

During the course of our studies of cyclopentadienyl(1,5cyclooctadiene)cobalt, CpCoCOD, we discovered that metal-

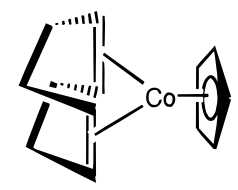

CpCoCOD

ligand stretching normal modes exhibit pronounced resonance deenhancements in the region of the lowest energy ligand field absorption band. The deenhancements are so striking that vibrational bands that are prominent at some excitation energies diminish to barely above the noise when the excitation is tuned to near the absorption maximum (Figure 1). Molecules of the type $\mathrm{CpM}$ (bis-alkene) are precursors for chemical vapor deposition; ${ }^{14-17} \mathrm{CpM}\left(\mathrm{C}_{2} \mathrm{H}_{4}\right)_{2}(\mathrm{M}=\mathrm{Rh}, \mathrm{Ir})$ have been used as precursors for laser-assisted chemical vapor deposition (LCVD) of pure metallic films. ${ }^{17}$ Assignments of the electronic excited states and knowledge of the bonding changes are needed in order

†Part of the special issue "Thomas Spiro Festschrift".

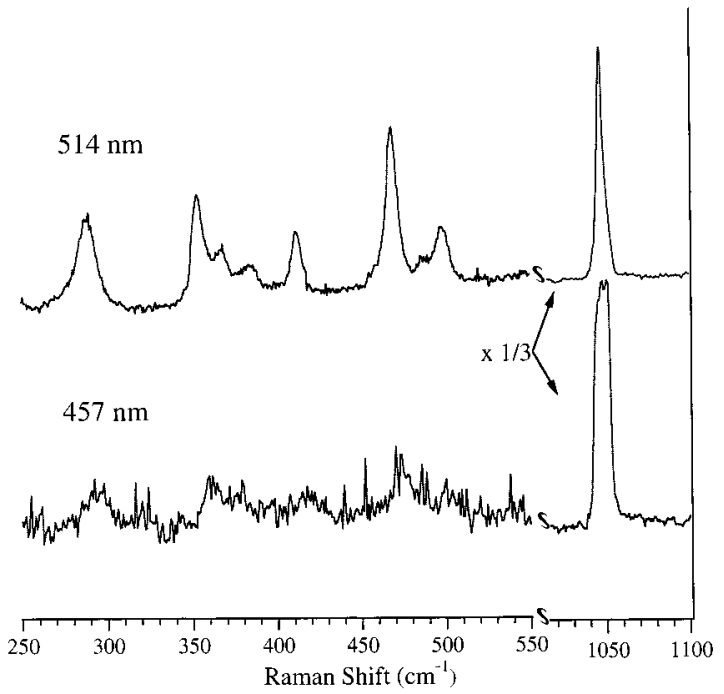

Figure 1. Raman spectra of CpCoCOD with (a) $514 \mathrm{~nm}$ and (b) 457 $\mathrm{nm}$ excitation. The peak at $1050 \mathrm{~cm}^{-1}$ is the $\mathrm{NO}_{3}{ }^{-}$reference peak and is shown on a scale $1 / 3$ that of the rest of the spectrum.

to understand the metal-ligand labilization process. The title compound in this study has an electronic structure analogous to the LCVD precursors, ${ }^{18}$ increased stability because of the bidentate diene ligand, and lower energy $d-d$ transitions that are separated from the charge-transfer (CT) transitions because of the first-row transition metal.

In this paper, we report the discovery of resonance Raman deenhancement in the excitation profiles of CpCoCOD. Deuteration studies are used to assign the metal-ligand stretching bands. The electronic excited states are assigned by measuring the single-crystal polarized electronic absorption spectra and using the results in conjunction with published molecular orbital calculations. ${ }^{18}$ The interfering excited states are identified. Excitation profiles are calculated by using the time-dependent theory of resonance deenhancement; ${ }^{12}$ the influences of multiple excited states and multiple normal modes are calculated and discussed. The origin of the interference effects and the physical 
meaning of the excited-state properties, including the signs of the distortions of the normal modes, are interpreted.

\section{Experimental Section}

(a) Materials. $\mathrm{CpCoCOD}, \mathrm{Cp}=$ cyclopentadienyl and COD $=$ cycloocta-1,5-diene, was prepared by literature procedures ${ }^{19}$ and purified by chromatography on neutral activated alumina with hexane as the eluent. The $\left(\eta^{5}-\mathrm{C}_{5} \mathrm{D}_{5}\right) \mathrm{CoCOD}$ was prepared by the same technique, using $\mathrm{C}_{5} \mathrm{D}_{6}$ instead of $\mathrm{C}_{5} \mathrm{H}_{6}{ }^{20}$ The extent of deuteration of the $\mathrm{Cp}$ ring was checked by NMR spectroscopy and found to be $>95 \%$.

(b) Spectroscopy. Solution absorption spectra of CpCoCOD in ethanol were obtained on a Shimadzu UV260 absorption spectrometer. Single-crystal polarized absorption spectra were obtained at room temperature at two orthogonal polarizations along the extinction directions of the crystals using an instrument described previously. ${ }^{21,22}$ The crystals were grown on quartz plates by slow evaporation of a saturated hexane solution. One drop of solution was placed on a quartz plate. The drop was allowed to evaporate over a $24 \mathrm{~h}$ period in an atmosphere of hexane. Several crystals usually formed on the plate; one that was well isolated from its neighbors was chosen for spectroscopy.

Resonance Raman spectra were obtained using either a Spex 1402 double monochromator equipped with an RCA C31034 photomultiplier tube and a Stanford Research Systems SR400 photon counter, or a Jobin-Yvon 320/640 triple monochromator equipped with a $256 \times 1024$ Princeton Instruments liquid nitrogen cooled CCD. Data were collected on a computer. The excitation source was a Coherent 90-6 argon ion laser, a Coherent 90-K krypton ion laser, or a UV pumped Spectra Physics dye laser. The excitation wavelengths used were 406 , 431, 441, 457, 488, 514, and $676 \mathrm{~nm}$. Scattered light was collected at a $90^{\circ}$ geometry. Peak locations are accurate to \pm 3 $\mathrm{cm}^{-1}$. The sample compound was mixed with $\mathrm{KNO}_{3}$, as an internal standard, in a 1:2.4 mass ratio.

\section{Results}

(a) Raman Spectra. Figure 1 shows Raman spectra of CpCoCOD obtained by using two different excitation wavelengths: $514 \mathrm{~nm}$ (at the leading edge of the lowest absorption band) and $457 \mathrm{~nm}$ (near the maximum of the lowest absorption band). The intensities of the peaks in the metal-ligand stretching region, from 300 to $500 \mathrm{~cm}^{-1}$, decrease relative to those of the peaks from the standard at 1050 and $712 \mathrm{~cm}^{-1}$ when the excitation wavelength is changed from 514 to $457 \mathrm{~nm}$.

A Raman spectrum of the deuterated compound, $\left(\eta^{5}-\mathrm{C}_{5} \mathrm{D}_{5}\right)$ CoCOD, was obtained to assist in assigning the vibrational bands. The $354 \mathrm{~cm}^{-1}$ band shifted to $338 \mathrm{~cm}^{-1}$ upon dueteration, a change of $16 \mathrm{~cm}^{-1}$ to lower frequency. The other metalligand bands shifted by smaller amounts. The $470 \mathrm{~cm}^{-1}$ band did not shift at all.

Table 1 lists all of the peaks with intensities of at least $10 \%$ of that of the most intense sample peak $\left(2985 \mathrm{~cm}^{-1}\right)$ observed in the $514 \mathrm{~nm}$ Raman spectrum. The modes at 290, 354, and $470 \mathrm{~cm}^{-1}$ have the largest decrease in intensity when the excitation frequency is changed from 514 to $457 \mathrm{~nm}$ excitation. The peaks that are grouped in section $\mathrm{b}$ of Table 1 show changes that are about the same as the experimental uncertainty. Those in section c do not change.

(b) Resonance Raman Spectroscopy. Raman excitation profiles were constructed in the region of the first electronic excited state to further quantify the relative intensity loss. In
TABLE 1. Raman Frequencies and Relative Intensities from 514 nm Excitation ${ }^{a}$

\begin{tabular}{|c|c|c|c|c|}
\hline & $\underset{\left(\mathrm{cm}^{-1}\right)}{\text { Raman shift }}$ & intensity & $\begin{array}{c} \pm \text { intensity } \\
\text { of peak }^{b}\end{array}$ & assignment \\
\hline \multirow[t]{3}{*}{$\mathrm{a}$} & 470 & 0.194 & 0.011 & \multirow{3}{*}{$\begin{array}{l}\mathrm{Co}-\mathrm{COD} \\
\mathrm{Co}-\mathrm{Cp} \\
\text { metal-ligand }\end{array}$} \\
\hline & 354 & 0.112 & 0.032 & \\
\hline & 290 & 0.112 & 0.001 & \\
\hline \multirow[t]{7}{*}{ b } & 3099 & 0.273 & 0.012 & \multirow{7}{*}{$\mathrm{CH}$} \\
\hline & 3082 & 0.112 & 0.035 & \\
\hline & 2985 & 0.347 & 0.040 & \\
\hline & 2971 & 0.105 & 0.019 & \\
\hline & 1233 & 0.049 & 0.002 & \\
\hline & 1108 & 0.296 & 0.006 & \\
\hline & 597 & 0.050 & 0.008 & \\
\hline \multirow[t]{7}{*}{$\mathrm{c}$} & 2867 & 0.136 & 0.009 & \multirow{7}{*}{\} $\mathrm{CH}$} \\
\hline & 2817 & 0.370 & 0.013 & \\
\hline & 1449 & 0.072 & 0.007 & \\
\hline & 1431 & 0.083 & 0.016 & \\
\hline & 776 & 0.046 & 0.003 & \\
\hline & 500 & 0.056 & 0.013 & \\
\hline & 412 & 0.071 & 0.004 & \\
\hline \multirow[t]{3}{*}{ d } & 1357 & 0.056 & 0.011 & \\
\hline & 1050 & 1.000 & 0.016 & \\
\hline & 712 & 0.064 & 0.003 & \\
\hline
\end{tabular}

${ }^{a}$ Section a, deenhancement pronounced; section b, deenhancement same magnitude within experimental uncertainty; section c, no deenhancement found; section $\mathrm{d}$, reference from $\mathrm{KNO}_{3} .{ }^{b}$ Average \pm 0.013 .

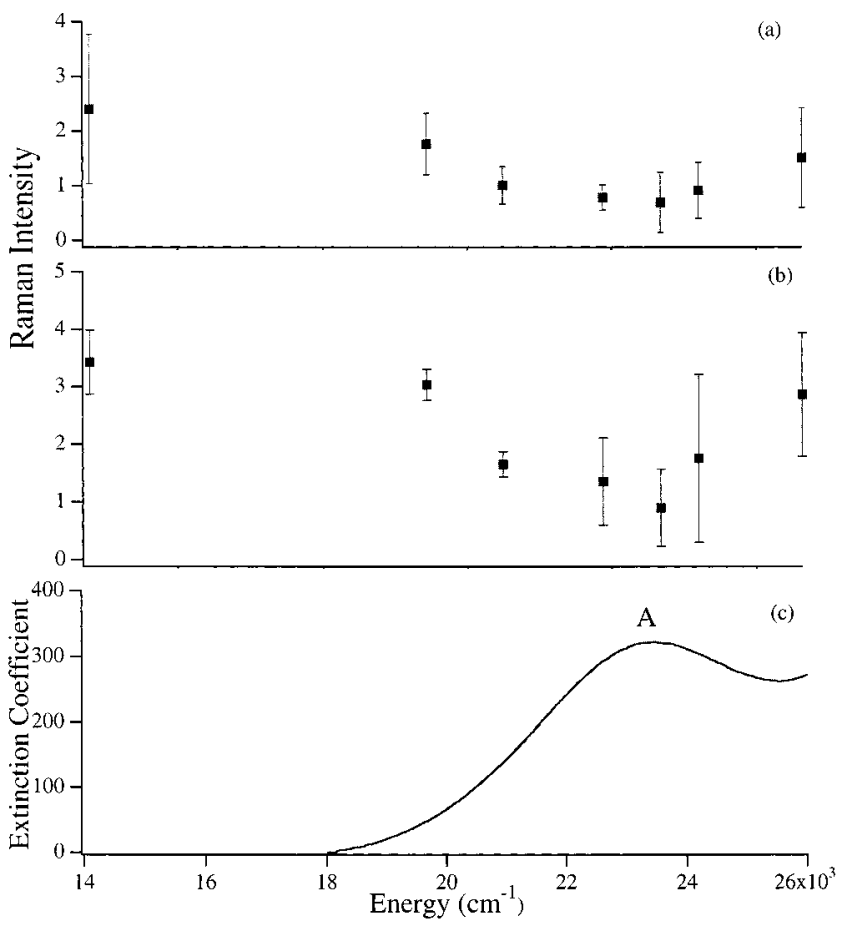

Figure 2. Resonance Raman excitation profiles for the $354 \mathrm{~cm}^{-1}$ (a) and $470 \mathrm{~cm}^{-1}$ (b) modes in the region of the lowest energy ligand field absorption band. The absorption spectrum (c) is shown in the bottom panel.

order to generate a resonance Raman excitation profile, it is necessary to employ an intensity standard that does not absorb in the range of excitation wavelengths used. $\mathrm{KNO}_{3}$ was chosen as the internal standard. It has NO peaks at 1050 and $712 \mathrm{~cm}^{-1}$ that are near the modes of interest in CpCoCOD.

The experimentally determined intensities of the 354 and 470 $\mathrm{cm}^{-1}$ peaks are shown in Figure 2 as a function of excitation energy (in wavenumbers). The absorption spectrum in the same energy region is included to highlight the position of the deenhancement in the region of the first absorption band. 


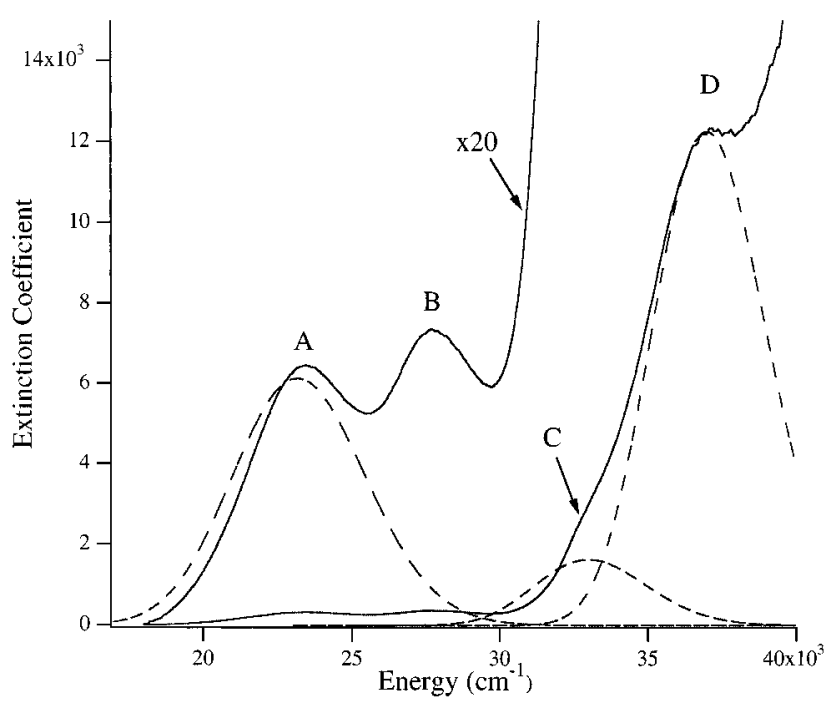

Figure 3. Absorption spectrum of $\mathrm{CpCoCOD}$ at room temperature $(0.1 \mathrm{mM}$ in ethanol). The calculated spectra, using the parameters in Table 2, are shown by broken lines.

The intensity values are corrected for self-absorption using the following formula

$$
\frac{I_{\mathrm{s}}}{I_{\mathrm{r} \text { corrected }}}=\frac{I_{\mathrm{s}}}{I_{\mathrm{r}}} \frac{c_{\mathrm{r}}}{c_{\mathrm{s}}} \frac{\left(\epsilon_{\mathrm{s}}+\epsilon_{0}\right)}{\left(\epsilon_{\mathrm{r}}+\epsilon_{0}\right)}
$$

where $c$ is the concentration of the reference or sample in the material tested and $\epsilon$ is the extinction coefficient of the sample at the reference, sample peak, or laser line (subscripts $r$, s, and 0 , respectively). ${ }^{23}$ All calculated intensities are compared to corrected intensities in the rest of the paper.

(c) Absorption Spectroscopy. The ethanol solution absorption spectrum of $\mathrm{CpCoCOD}$ at room temperature is shown in Figure 3. The spectrum contains bands at 23450 and 27700 $\mathrm{cm}^{-1}$, labeled $\mathrm{A}$ and $\mathrm{B}$, respectively, along with several overlapping bands at higher energy. Of these higher energy bands, the shoulder at $33050 \mathrm{~cm}^{-1}$ and peak at $37100 \mathrm{~cm}^{-1}$, labeled $\mathrm{C}$ and $\mathrm{D}$, will play a role in the following discussion. Bands A and B have extinction coefficients of 320 and 370 $\mathrm{M}^{-1} \mathrm{~cm}^{-1}$, while those of $\mathrm{C}$ and D are 3000 and $12300 \mathrm{M}^{-1}$ $\mathrm{cm}^{-1}$, respectively.

Single-crystal absorption measurements were made using rectangular microcrystals. The extinction axes are along the long and short axes of the crystal. Figure 4 shows the single-crystal polarized absorption spectra of CpCoCOD. Data are presented for two orthogonal polarization directions parallel and perpendicular to the long axis of the crystal. The peaks are labeled in the same manner as those in the solution absorption spectrum. The two low-energy transitions are of different polarizations. Peak B is present in the perpendicular polarization but is absent in the parallel polarization. Peak A is prominent in the parallel but is barely visible in the perpendicular polarization.

\section{Discussion}

The resonance Raman excitation profile for $\mathrm{CpCoCOD}$ in the wavenumber region between 15000 and $24000 \mathrm{~cm}^{-1}$ contains a decrease in intensity or deenhancement with its minimum near $23000 \mathrm{~cm}^{-1}$. In this same region, the absorption spectrum contains peak A, with its maximum at about the same energy as that of the deenhancement minimum. The behavior of the excitation profile is unusual; normally, a maximum in the profile is found at the maximum of an absorption band. Two

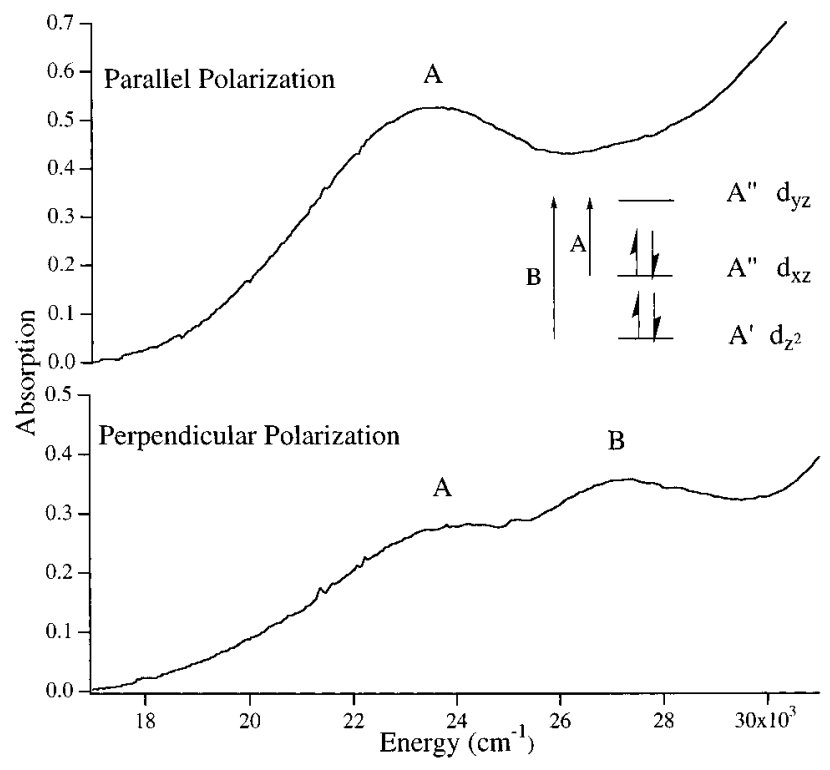

Figure 4. Single-crystal polarized absorption spectra of CpCoCOD in orthogonal polarization directions. A partial molecular orbital diagram is shown in the inset.

of the deenhancement profiles and the absorption spectrum are shown on the same scale in Figure 2.

The theory of resonance deenhancement has recently been developed..$^{11-13}$ The intensity of a resonance Raman peak is proportional to the square of the scattering cross section $\alpha^{24-26}$ The scattering cross section, in turn, is related to the square of the transition dipole moment and thus usually follows the absorption spectrum. However, if there is another electronic state nearby, the Raman intensity is proportional to the square of the sum of the cross sections. If the cross sections are of opposite sign, destructive interference can occur, resulting in resonance deenhancement. ${ }^{24-26}$

The solution absorption spectra of CpCoCOD contain three absorption bands near the region of band $\mathrm{A}$ where the deenhancement occurs. Any or all of them could provide the source of the interference if the magnitude of $\alpha$ from them is similar to that of band A at $23000 \mathrm{~cm}^{-1}$ and destructive interference occurs when the sum of the cross sections is squared. In the following section, the electronic states and the normal modes involved in the interference are assigned and the deenhancement is quantitatively calculated.

(a) Assignment of Absorption Bands. The bands at 23450 and $27700 \mathrm{~cm}^{-1}$ have extinction coefficients of about $300 \mathrm{M}^{-1}$ $\mathrm{cm}^{-1}$, while those of the band at $37100 \mathrm{~cm}^{-1}$ and shoulder at $33050 \mathrm{~cm}^{-1}$ are 12000 and $3000 \mathrm{M}^{-1} \mathrm{~cm}^{-1}$, respectively. These values suggest that the former are $d-d$ in character, while the latter are charge-transfer.

A molecular orbital calculation for CpCoCOD has been published along with orbital energies obtained from XPS spectroscopy. ${ }^{18}$ The important portion of the MO diagram is shown in the inset of Figure 4. The labeled orbitals are primarily metal-d in character, with the specific d orbitals indicated. The labels of the $d_{x z}$ and $d_{y z}$ orbitals depend on the choice of the coordinate system; in this paper, the $x$-axis is defined as the axis parallel to both the cyclopentadiene ring and the carboncarbon double bonds of the COD ring, while the $y$-axis is defined as the axis parallel to the cyclopentadiene ring and perpendicular to the COD double bonds. Orbital energies were reported only for the occupied orbitals. However, the energy difference between the highest and second highest occupied orbitals $(0.58$ $\mathrm{eV}$ ) agrees with the observed energy difference between the 


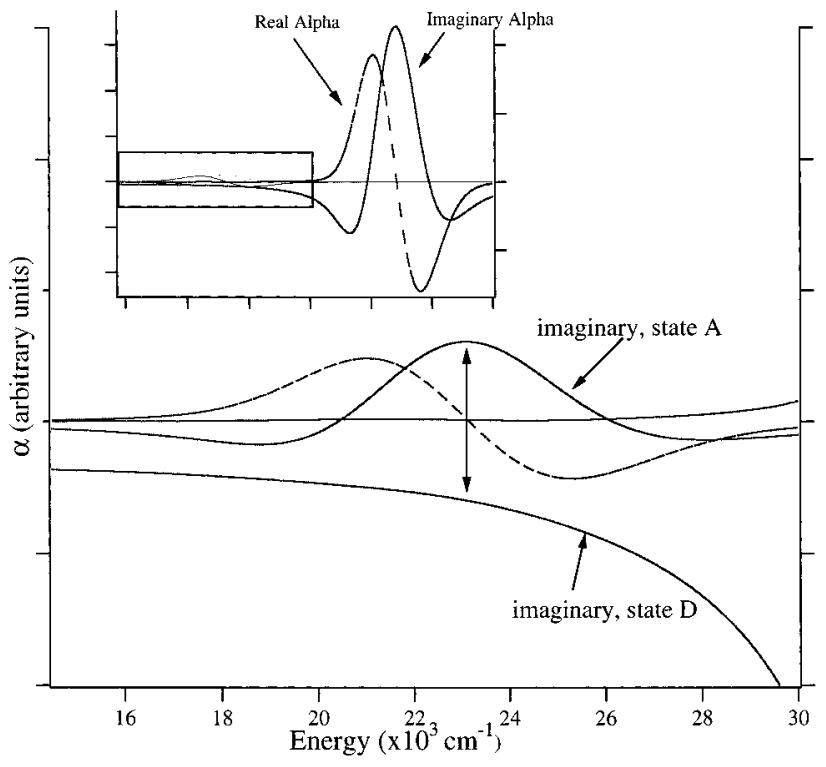

Figure 5. Real (broken line) and imaginary (solid line) components of the resonance Raman scattering cross section $\alpha$ for two excited electronic states. The cancellation of the imaginary components at the wavenumber of the lowest ligand field state is shown by the doubleheaded arrow. The inset shows the components of the interfering chargetransfer state. The region enclosed by the rectangle in the inset is magnified in the main figure. The parameters are given in Table 2.

360 and $430 \mathrm{~nm}$ absorption bands $(0.56 \mathrm{eV})$. We therefore assign the lowest energy band (A) as $\mathrm{d}_{x z} \rightarrow \mathrm{d}_{y z}$ and the middle energy band (B) as $\mathrm{d}_{z^{2}} \rightarrow \mathrm{d}_{y z}$.

These assignments are supported by the polarized absorption data that show that the two lowest energy absorption bands are of different polarizations. The ground state of the molecule $\left(C_{s}\right.$ point group) is $\mathrm{A}^{\prime}$. The excited state corresponding to the lowest energy band is also $\mathrm{A}^{\prime}$, so the transition is z-polarized. The excited state corresponding to the middle energy band is $\mathrm{A}^{\prime \prime}$, so the transition is $x, y$-polarized.

From the MO calculations, the lowest energy charge-transfer transition originates from a one-electron transition from the $\mathrm{d}_{x z}$ HOMO to the second-lowest unoccupied orbital. This transition is metal-to-COD in nature, with the electron entering a $\pi^{*}$ orbital on the diene ligand.

(b) Assignment of Metal-Ligand Raman Bands. The assignments of the 354 and $470 \mathrm{~cm}^{-1}$ vibrations can be determined by comparison of the Raman spectrum of CpCoCOD with that of the dueterated $\mathrm{Cp}$ analogue. Since only the cyclopentadienyl ring is deuterated in the analogue, comparison of the vibrational frequencies allows for discrimination of $\mathrm{M}-\mathrm{Cp}$ modes from $\mathrm{M}-\mathrm{COD}$ modes. The $354 \mathrm{~cm}^{-1}$ mode shifts by $16 \mathrm{~cm}^{-1}$ to lower frequency, while the $470 \mathrm{~cm}^{-1}$ mode does not shift at all. The $354 \mathrm{~cm}^{-1}$ peak is therefore inferred to be primarily a $\mathrm{Co}-\mathrm{Cp}$ vibration, while the $470 \mathrm{~cm}^{-1}$ mode is primarily Co-COD. The former assignment is consistent with the literature value of $355 \mathrm{~cm}^{-1}$ for the $\mathrm{Co}-\mathrm{Cp}$ stretching mode of cobaltocene. ${ }^{27}$ The other metal-ligand peaks most likely arise from vibrations involving both ligands. Since their frequencies shift with deuteration of the Cp ring, some motion of this ligand must be involved. However, the smaller shifts relative to that of the $354 \mathrm{~cm}^{-1}$ mode suggest that other components also contribute to the normal coordinate.

(c) Theory of Resonance Deenhancement. The resonance Raman excitation profiles are calculated by using the timedependent theory of spectroscopy, including interference between multiple electronic states. ${ }^{12,13,28}$ Interference between states is interpreted by examining the constructive or destructive interference between the real and imaginary parts of the Raman scattering cross section. The resonance Raman intensity for $n$ excited states is given by

$$
I_{\mathrm{i}-\mathrm{f}} \propto\left|\sum_{r=1}^{n} \alpha_{r}\right|^{2}
$$

The scattering cross section $\alpha$ for the $r$ th electronic state is given by

$$
\alpha_{r}=\frac{i}{\hbar} \int_{0}^{\infty}\left\langle\phi_{\mathrm{f}} \mid \phi(t)\right\rangle_{r} \exp \left\{i\left(\omega_{\mathrm{i}}+\omega_{\mathrm{I}}\right) t\right\} \mathrm{d} t
$$

The overlap function includes $\left\langle\phi_{\mathrm{f}}\right|=\left\langle\chi_{\mathrm{f}}\right| \mu$, where $\chi_{\mathrm{f}}$ is the final vibrational state of the ground electronic surface multiplied by the transition dipole moment $\mu ;|\phi\rangle=\mu|\chi\rangle$ is the electric transition dipole moment multiplied by the initial vibrational state of the ground electronic surface. The zero-point energy of the ground-state electronic surface and the energy of the incident radiation are $\hbar \omega_{\mathrm{i}}$ and $\hbar \omega_{\mathrm{I}}$, respectively. The time-dependent overlap for a given electronic excited state $r$ of the final function $\phi_{\mathrm{f}}$ with the propagating wave packet $\phi(t)$ is given by

$$
\begin{aligned}
&\left\langle\phi_{\mathrm{f}} \mid \phi(t)\right\rangle_{r}= \\
& \mu_{r}^{2} \prod_{k}\left\{\exp \left[-\frac{\Delta_{k}^{2}}{2}\left(1-\exp \left(-\mathrm{i} \omega_{k} t\right)\right)-\frac{i \omega_{k} t}{2}\right](1-\right. \\
&\left.\left.\quad \exp \left(-i \omega_{k} t\right)\right)^{n_{k}} \frac{(-1)^{n_{k}} \Delta_{k}^{n_{k}}}{\left(2^{n_{k}} n_{k} !\right)^{1 / 2}}\right\} \exp \left(-i \omega_{0_{\mathrm{r}}} t-\Gamma_{r} t\right)
\end{aligned}
$$

The transition dipole moment for the $r$ th excited state is $\mu_{r}$. The energy of the zero-zero transition to the $r$ th excited state is $\omega_{0_{\mathrm{r}}}$, in inverse centimeters. The vibrational frequency $\left(\mathrm{cm}^{-1}\right)$ and the displacement of the $k$ th normal mode in the $r$ th excited state are $\omega_{k}$ and $\Delta_{k}$, respectively. The vibrational quantum number of the $k$ th normal mode in the ground state is $n_{k}$. The damping factor for the $r$ th excited state is $\Gamma_{r}$.

The equation for the time-dependent overlap shown in eq 4 assumes that (a) the potential surfaces are harmonic, (b) the normal coordinates are not mixed in the excited state, (c) the transition dipole moment is coordinate independent, and (d) the force constants do not change in the excited state.

The transition dipole acts as a scaling factor that determines the contribution of the $r$ th excited-state overlap to the total resonance Raman cross section. It is obtained from the absorption spectrum. The overlap, $\langle\phi \mid \phi(t)\rangle$, consists of real and imaginary parts. The real or imaginary parts for two electronic states may be of equal magnitude but opposite sign at a given wavenumber, and the resulting profile may not match the electronic spectrum in that region. The imaginary component of $\alpha$ is negative at the low wavenumber side of the absorption maximum when $\Delta$ is positive. Figure 5 shows the real and imaginary components for two excited states. In the region where an excited state with a small transition dipole moment has an imaginary $\alpha$ that coincides with the tail of the imaginary component of an excited state with a much larger transition dipole moment, cancellation of components is observed.

Additional electronic states may contribute to the deenhancement if the signs and magnitudes of $\alpha$ are appropriate. The bigger the energy separation, the greater the value of $\mu$ necessary to give a magnitude appropriate for interference. 
TABLE 2. Values of Parameters Used in the Calculations

\begin{tabular}{|c|c|c|c|}
\hline $\begin{array}{l}\text { parameters for 2-state, } \\
\text { 2-mode calc }\end{array}$ & \multicolumn{2}{|c|}{$\begin{array}{c}\text { state A, location of } \\
\text { deenhancement }\end{array}$} & state D \\
\hline$E_{00}\left(\mathrm{~cm}^{-1}\right)$ & \multicolumn{2}{|l|}{15000} & 29000 \\
\hline$\Gamma\left(\mathrm{cm}^{-1}\right)$ & \multicolumn{2}{|l|}{850} & 200 \\
\hline$\Delta_{354}$ & \multicolumn{2}{|l|}{3.9} & 3.9 \\
\hline$\Delta_{470}$ & \multicolumn{2}{|l|}{4.8} & 4.8 \\
\hline$\mu^{2}$ & \multicolumn{2}{|l|}{1} & 40 \\
\hline $\begin{array}{c}\text { parameters for 2-state, } \\
\text { 3-mode calc }\end{array}$ & \multicolumn{2}{|c|}{$\begin{array}{c}\text { state A, location of } \\
\text { deenhancement }\end{array}$} & state D \\
\hline$E_{00}\left(\mathrm{~cm}^{-1}\right)$ & \multicolumn{2}{|l|}{15000} & 29000 \\
\hline$\Gamma\left(\mathrm{cm}^{-1}\right)$ & \multicolumn{2}{|l|}{550} & 200 \\
\hline$\Delta_{290}$ & \multicolumn{2}{|l|}{3.75} & 3.75 \\
\hline$\Delta_{354}$ & \multicolumn{2}{|l|}{3.25} & 3.25 \\
\hline$\Delta_{470}$ & \multicolumn{2}{|l|}{4.25} & 4.25 \\
\hline$\mu^{2}$ & \multicolumn{2}{|l|}{1} & 40 \\
\hline $\begin{array}{l}\text { parameters for 3-state, } \\
\text { 2-mode calc }\end{array}$ & $\begin{array}{c}\text { state A, location of } \\
\text { deenhancement }\end{array}$ & state D & state $\mathrm{C}$ \\
\hline$E_{00}\left(\mathrm{~cm}^{-1}\right)$ & 15000 & 29000 & 25000 \\
\hline$\Gamma\left(\mathrm{cm}^{-1}\right)$ & 850 & 200 & 100 \\
\hline$\Delta_{354}$ & 3.9 & 3.9 & 3.9 \\
\hline$\Delta_{470}$ & 4.8 & 4.8 & 4.8 \\
\hline$\mu^{2}$ & 1 & 40 & 7.5 \\
\hline
\end{tabular}

(d) Calculation of Deenhancement Profiles Using Two Electronic States. The deenhancements occur near the maximum of the $d_{x z} \rightarrow d_{y z}$ band A; the imaginary components of $\alpha$ from this ligand field excited state are obviously involved. As an initial choice for the interfering excited state, the chargetransfer state with the largest molar absorptivity, band D, is tested. The negative part of $\alpha$ has about the same magnitude as the positive imaginary part from state A, as shown in Figure 5.

The best fit to the excitation profiles of the 354 and $470 \mathrm{~cm}^{-1}$ normal modes is shown in Figure 6A and $\mathrm{B}$. The values of $\mu$, $\Delta, E_{00}$, and $\Gamma$ that are used in the calculations are given in Table 2. Note that calculations involving multiple normal modes must simultaneously include all of the modes (eq 2). Furthermore, the fit to the excitation profiles is subject to limiting constraints, because all of the Raman intensities and experimental absorption spectra (Figure 3) must be accurately calculated.

There is an interaction between the values of $\mu, \Delta, E_{00}$, and $\Gamma$ that can be used to obtain a fit; changing one of them may sometimes be compensated for by a change in another. However, these changes can only be made over a narrow range and still retain a good fit to both the absorption and Raman spectra. The value of $\mu$ is critical to the calculation of the Raman intensities, but its value is fixed by the absorption spectrum. Similar considerations apply to $E_{00}$. The magnitude of $\Gamma$ cannot be determined independently in this case of interfering states. The values that are used in the fit are well within the range that has been used for metal complexes and are small enough that they do not artificially contribute to the absorption line width. The range of $\Gamma$ that produced a good fit was $\pm 100 \mathrm{~cm}^{-1}$.

The most interesting and important components of the fit are the values of the distortion $\Delta$. These values can only be varied over a small range and still maintain a good fit of both the Raman excitation profiles and absorption data. In the case of the two-vibrational normal mode fit discussed here, the fit became significantly poorer and unacceptable when the $\Delta$ 's were increased or decreased by $10 \%$ for the $d-d$ state and $20 \%$ for the charge-transfer state.

The relative signs of the distortions can be determined from the fit. In the usual excitation profiles where only one electronic state contributes, only the magnitude can be calculated. However, when two states interfere, the signs of the distortions affect
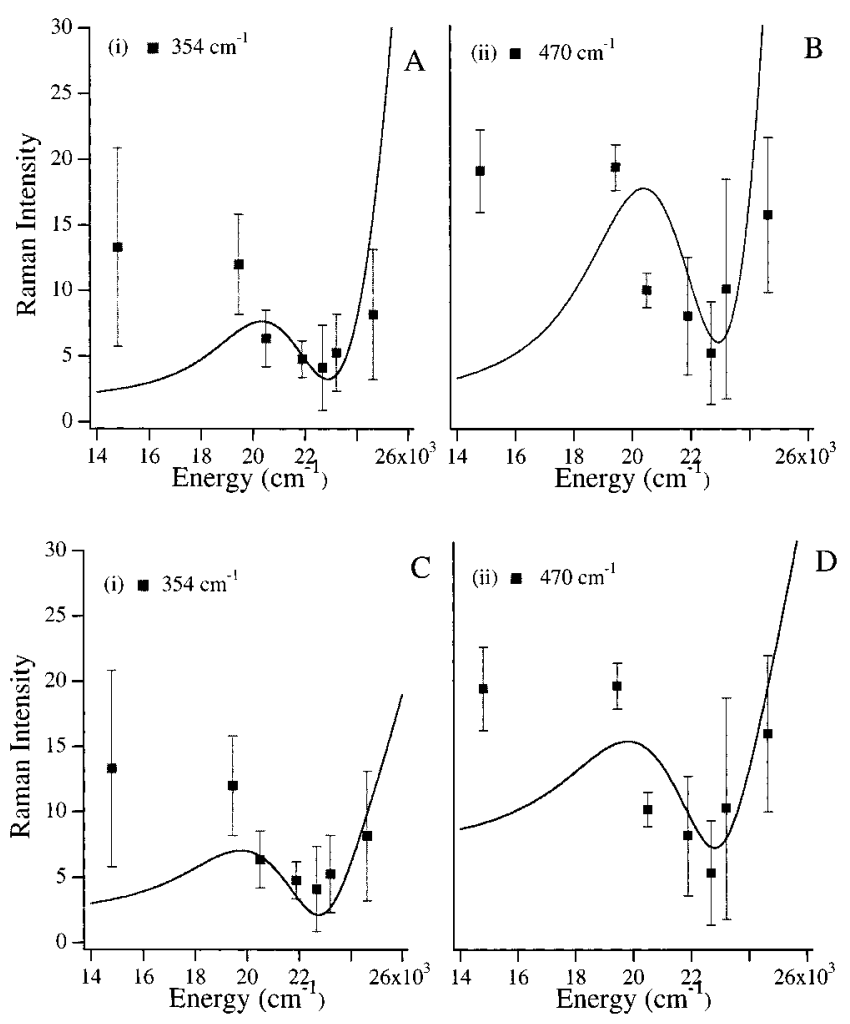

Figure 6. Experimental (squares) and calculated (lines) resonance Raman excitation profiles for the $354 \mathrm{~cm}^{-1}$ (i) and $470 \mathrm{~cm}^{-1}$ (ii) modes. The experimental data are corrected for self-absorption. The top two panels show the results for two states, $\mathrm{A}$ and $\mathrm{D}$, and the bottom two panels show the results for three states, A, C, and D. The parameters used in the calculations are given in Table 2 .

the signs of the real and imaginary components of $\alpha$ that, in turn, affect whether destructive interference occurs. A negative distortion in the $\mathrm{d}-\mathrm{d}$ state will change the sign of the components of $\alpha$ in Figure 5 and result in enhancement rather than deenhancement. Because deenhancement is observed in both of the modes calculated in Figure 6, the sign of the distortion must be the same in both states for each mode.

Deenhancement from the Lowest Energy CT State. The absorption spectrum shows a shoulder on the low-energy side of the large charge-transfer band. This state, labeled $\mathrm{C}$, has a smaller extinction coefficient and is nearer in energy to the $\mathrm{d}-\mathrm{d}$ state than the large charge-transfer state $\mathrm{D}$. To cause interference of the imaginary parts, this smaller ratio of transition dipoles is exactly what is required of a state that is closer in energy. Calculations containing states $\mathrm{A}$ and $\mathrm{C}$ produce a deenhancement in the region of the $d-d$ state, A. State $C$ must obviously be included in a more complete model; a three-state calculation with states $\mathrm{A}, \mathrm{C}$, and $\mathrm{D}$ is discussed in section $\mathrm{E}$ below.

Absence of Interference from the Other $d-d$ State. The second $\mathrm{d}-\mathrm{d}$ transition, $\mathrm{B}$, does not contribute to the deenhancement. The transition dipoles of the two states are similar, and the states are sufficiently separated in energy such that the imaginary part from state B does not cancel out that from state A. In fact, the calculated Raman excitation profiles for states A and B are similar to the solution absorption spectrum in the same region.

Deenhancement of Three Modes. Three modes show significant deenhancement in the experimental profiles. Additional modes can easily be included in the calculation (eq 4). Inclusion of additional modes that do not show deenhancement acts like the damping factor and does not significantly change the results.

To demonstrate the effect of additional modes, the $290 \mathrm{~cm}^{-1}$ mode (that does show deenhancement) is included. The 

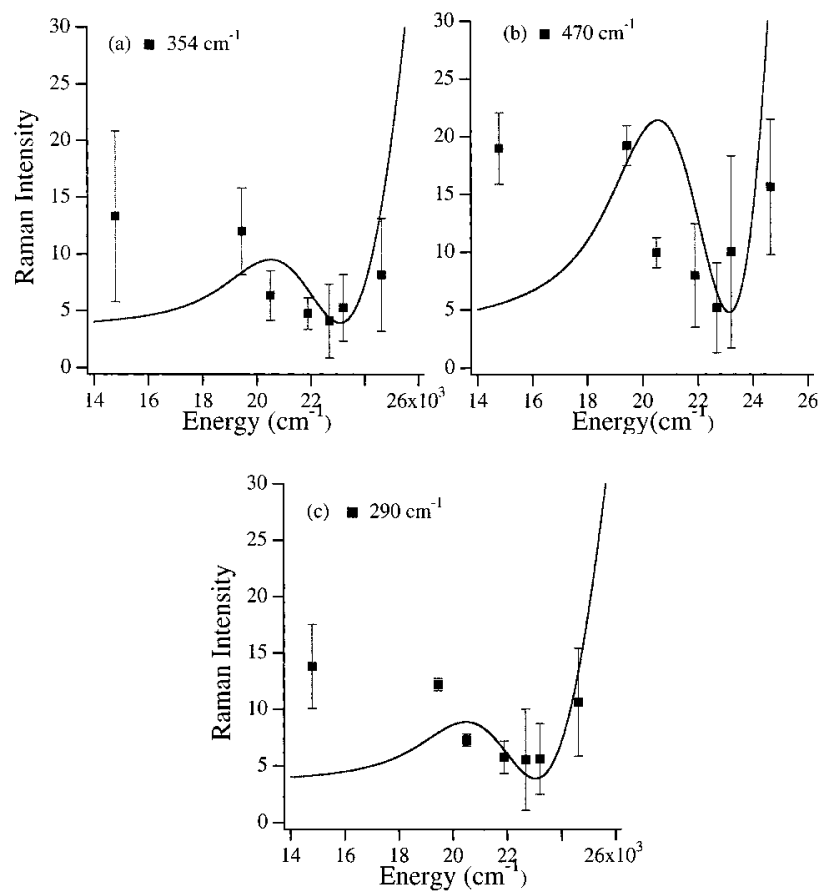

Figure 7. Experimental (squares) and calculated (lines) resonance Raman excitation profiles for the $354 \mathrm{~cm}^{-1}$ (a), $470 \mathrm{~cm}^{-1}$ (b), and 290 $\mathrm{cm}^{-1}$ (c) modes. The calculations include all three modes and two states, $\mathrm{A}$ and $\mathrm{D}$. The parameters used in the calculations are given in Table 2.

calculated deenhancement profiles for the three-mode calculation are shown in Figure 7; the parameters are in Table 2.

(e) Calculation of Deenhancement Profiles Using Three Electronic States. From the two state calculations, both of the charge-transfer states $\mathrm{C}$ and D can contribute to the deenhancement. The time-dependent theory allows for the inclusion of multiple states in the Raman intensity through the sum of the Raman cross sections, as shown in eq 2 .

$E_{00}, \mu$, and $\Gamma$ are constrained by the absorption spectra, as discussed above. In order to give an illustrative calculation, the $\Delta$ parameters for states $\mathrm{C}$ and $\mathrm{D}$ are chosen to be the same. The calculated Raman excitation profiles are shown in Figure $6 \mathrm{C}$ and D.

Even though the second $d-d$ transition cannot be the origin of the deenhancement, it could be added as a fourth state in the calculations to further modify the fit. However, inclusion of four states requires an unreasonable number of variables, and the meaning of the parameters becomes less certain.

\section{Physical Meaning}

(a) Signs of the Distortions. When the overlap of two or more absorption bands causes interference leading to resonance deenhancement in the Raman excitation profile, the bond length changes that occur as a result of populating the first state cannot be determined independently from those of the second state. Because they must be simultaneously calculated, the relative signs and not just the absolute values of the distortions can be determined. Thus, the presence of the deenhancement can be an advantage in the analysis of excited distortions because it provides a method of calculating the relative distortions of two states in a molecule.

The calculation of the deenhancement in CpCoCOD requires that the signs of the distortions of the $354 \mathrm{~cm}^{-1} \mathrm{Co}-\mathrm{Cp}$ stretch, the $470 \mathrm{~cm}^{-1} \mathrm{Co}-\mathrm{COD}$ stretch, and the $290 \mathrm{~cm}^{-1}$ metal-ligand stretching modes are the same in all of the excited states. It does not specify positive or negative; the distortions could all be positive (bond lengthening) or negative (bond contracting). The signs of the distortions can be inferred on the basis of the results of the molecular orbital calculation and the identity of the MO's that are involved in the transitions. The $d-d$ transition involves the promotion of an electron to a metal-based antibonding molecular orbital. The increase in antibonding will decrease the bond order between the metal and ligand, lengthening the bond in the excited state. Thus, the signs of the metalligand distortions in the $\mathrm{d}-\mathrm{d}$ excited state are probably positive. In the charge-transfer excited state, the electron is promoted from a metal-ligand bonding orbital to a ligand-centered orbital that is not involved in bonding to the metal. Because the electron is removed from a bonding orbital, the net bonding between the metal and ligands is decreased, bond elongation should occur, and the signs of all of the metal-ligand distortions are also expected to be positive. This reasoning is in agreement with the calculated requirement that the distortions have the same sign in both states and suggests that the sign is positive.

(b) Magnitudes of the Bond Length Changes. The distortions given in Table 2 are dimensionless distortions. Conversion to angstrom units provides more physical meaning to these distortions. The conversion is

$$
\delta=10^{8} \Delta \sqrt{\frac{6.023 \times 10^{23}}{m} \frac{\hbar}{2 \pi c \omega}}
$$

where $\delta$ is the bond length change in angstroms, $\Delta$ is the dimensionless distortion, $\omega$ is the frequency of the mode, $m$ is the mass involved, $\hbar$ is Plank's constant divided by $2 \pi$, and $c$ is the speed of light.

In order to accurately calculate the distortion of a given bond, a normal coordinate analysis is necessary. ${ }^{29}$ However, a rough estimate can be made if simplifying assumptions about the normal masses and potential energy distribution are made. In the case of the $470 \mathrm{~cm}^{-1}$ mode, a simplifying assumption is that the motion is predominantly $\mathrm{Co}-\mathrm{COD}$ in character and the mass is that of two "ethylene" units. In the case of the 354 $\mathrm{cm}^{-1}$ mode, it is assumed that the motion is prominently $\mathrm{Co}-$ $\mathrm{Cp}$ in character and the mass is the mass of the $\mathrm{Cp}$ ligand.

The bond length change in the $\mathrm{d}-\mathrm{d}$ transition excited state will be about $0.10 \AA$ for the $\mathrm{Co}-\mathrm{COD}$ distance ${ }^{30}$ and $0.14 \AA$ for the $\mathrm{Co}-\mathrm{Cp}$ distance. These estimates are upper limits because the actual normal modes include motions other than pure $\mathrm{Co}-\mathrm{Cp}$ and $\mathrm{Co}-\mathrm{COD}$ stretches. These are similar to values determined for bond length changes in other $\mathrm{M}-\mathrm{C}_{2} \mathrm{H}_{4}$ and $\mathrm{M}-\mathrm{Cp}$ moieties in $\mathrm{d}-\mathrm{d}$ transitions. The $\mathrm{M}-\mathrm{Cp}$ bond length change in ruthenocene is $0.12 \AA .{ }^{31}$ The change in the metalolefin distance for Zeise's salt is $0.18 \AA .{ }^{32}$

The calculated trends in the bond length changes are consistent with the assignments of the excited state. In the case of the $\mathrm{d}-\mathrm{d}$ excited state, both the HOMO and the LUMO are primarily metal $\mathrm{d}$ in character with both $\mathrm{Cp}$ and $\mathrm{COD} \pi$ antibonding components. The lowest energy $d-d$ transition should therefore involve simultaneous lengthening of both metal-ligand bonds, as is found by the fit to the deenhancement profile.

\section{Summary}

The pronounced resonance deenhancement in the excitation profiles of the $\mathrm{Co}-\mathrm{Cp}$ and $\mathrm{Co}-\mathrm{COD}$ metal-ligand stretching modes is caused by interference between the lowest energy $\mathrm{d}_{x z}$ $\rightarrow \mathrm{d}_{y z}$ ligand field excited state and $\mathrm{Co} \rightarrow \mathrm{COD}$ metal-ligand charge-transfer excited states. The imaginary part of the Raman scattering cross section from the ligand field state has the same 
magnitude but opposite sign of those from the charge-transfer states. The intensity of the bands in the Raman spectrum is proportional to the square of the sum of the cross sections. The greatest interference occurs near the region of the maximum of the absorption band of the state with the smallest cross section. The relative signs of the imaginary parts are dependent on the relative signs of the displacements of the potential surfaces. Thus, the presence of the interference enables the relative signs to be calculated. The metal-ligand bonds in both the ligand field and the charge-transfer states are distorted in the same direction according to the fit; molecular orbital calculations suggest that both of the bonds are elongated. Good fits to the deenhancement profiles and the absorption spectra are obtained by including one or both of the nearby charge-transfer states.

The parameters that are used in the calculations are constrained. The electronic origins $\left(E_{00}\right)$ and the transition dipole moments $(\mu)$ of the electronic states are fixed and obtained from the absorption spectrum. The distortions of the normal modes $(\Delta)$ can only be varied by about $\pm 10 \%$ without significantly degrading the fit. The displacements of the normal coordinates can be converted to individual metal-ligand bond length changes if the normal coordinates and potential energy distributions are known; in their absence, a rough estimate of the masses suggests that the metal-Cp bond changes by $0.14 \pm 0.02 \AA$ and the metal-olefin bond by $0.10 \pm 0.01 \AA$.

When a second state is present, there is always some interference, although the contribution may be so small that it will not noticeably affect the shape or location of important features in the spectra. However, if a second state is implicated as the origin of differences between the absorption spectrum and Raman excitation profile, then the parameters for the two states must be determined simultaneously.

Acknowledgment. This work was made possible by a grant from the National Science Foundation (CHE9816552). We thank Dr. Alf Bacher and Andy Cerin for assistance with the syntheses, and Prof. Derk Stufkens and Th. L. Snoeck for assistance with the dye laser Raman measurements.

\section{References and Notes}

(1) Zink, J. I.; Shin, K. S. K. Advances in Photochemistry; Wiley: New York, 1991; Vol. 16, p 119.
(2) Myers, A. B. In Laser Techniques in Chemistry; Myers, A. B., Rizzo, T. R., Eds.; Wiley: New York, 1995; p 325.

(3) Shin, K. S. K.; Zink, J. I. Inorg. Chem. 1989, 28, 4358.

(4) Henary, M.; Wootton, J. L.; Khan, S. I.; Zink, J. I. Inorg. Chem. 1997, 36, 796.

(5) Shin, K. S. K.; Clark, R. J. H.; Zink, J. I. J. Am. Chem. Soc. 1990, 112,3754 .

(6) Shin, K. S.; Clark, R. J. H.; Zink, J. I. J. Am. Chem. Soc. 1989, $111,4244$.

(7) Nafie, L. A.; Pastor, R. W.; Dabrowiak, J. C.; Woodruff, W. H. J. Am. Chem. Soc. 1976, 98, 8007.

(8) Schick, G. A.; Bocian, D. F. J. Raman Spectrosc. 1981, 11, 27.

(9) Fodor, S. P. A.; Copeland, R. A.; Grygon, C. A.; Spiro, T. G. J. Am. Chem. Soc. 1989, 111, 5509.

(10) Ribeiro, M. C. C.; Oliveira, L. F. C.; Constantino, V. R. L.; Toma, H. E.; Santos, P. S. J. Raman Spectrosc. 1993, 24, 431.

(11) Stein, P.; Miskowski, V.; Woodruff, W. H.; Griffin, J. P.; Werner,

K. G.; Gaber, B. P.; Spiro, T. G. J. Chem. Phys. 1976, 64, 2159.

(12) Shin, K. S. K.; Zink, J. I. J. Am. Chem. Soc. 1990, 112, 7148.

(13) Wootton, J. L.; Zink, J. I. J. Am. Chem. Soc. 1997, 119, 1895.

(14) Kaesz, H. D.; Williams, R. S.; Hicks, R. F.; Zink, J. I.; Chen, Y.J.; Müller, H.-J.; Xue, Z.; Xu, D.; Shuh, D. K.; Kim, Y. K. New J. Chem. 1990, 14, 527.

(15) Chen, Y. J.; Kaesz, H. D.; Thridandam, H.; Hicks, R. F. Appl. Phys. Lett. 1988, 53, 1591 .

(16) Pierson, H. O. Handbook of Chemical Vapor Deposition: Principles, Technology and Applications; Noyes Publications: Westwood, NJ, 1992

(17) Cohan, J. S.; Yuan, H.; Williams, R. S.; Zink, J. I. Appl. Phys. Lett. 1992, 60, 1402 .

(18) Green, J. C.; Powell, P.; van Tilborg, J. E. Organometallics 1984, $3,211$.

(19) Bönneman, H.; Bogdanovic, B.; Brinkman, R.; He, D. W.; Spliethoff, B. Angew. Chem., Int. Ed. Engl. 1983, 22, 728.

(20) Lambert, J. B.; Finzel, R. B. J. Am. Chem. Soc. 1983, 105, 1954.

(21) Chang, T. H.; Zink, J. I. J. Am. Chem. Soc. 1986, 108, 5830.

(22) Reber, C.; Zink, J. I. Inorg. Chem. 1991, 30, 2994.

(23) Shriver, D. F.; Dunn, J. B. R. Appl. Spectrosc. 1974, 28, 319.

(24) Lee, S. Y.; Heller, E. J. J. Chem. Phys. 1979, 71, 4777.

(25) Heller, E. J.; Sundberg, R. L.; Tannor, D. J. Phys. Chem. 1982, $86,1822$.

(26) Tannor, D.; Heller, E. J. J. Chem. Phys. 1982, 77, 202.

(27) Nakamoto, K. Infrared and Raman Spectra of Inorganic and Coordination Compounds; Wiley-Interscience: New York, 1978.

(28) Reber, C.; Zink, J. I. J. Phys. Chem. 1992, 96, 571.

(29) Wootton, J. L.; Zink, J. I.; Fleming, G. D.; Vallette, M. C. Inorg. Chem. 1997, 36, 789.

(30) The normal coordinate is $1 / \sqrt{2}\left(\Delta d_{1}+\Delta d_{2}\right)$ when $\Delta d$ is the Coolefin displacement.

(31) Hollingsworth, G. J.; Shin, K. S.; Zink, J. I. Inorg. Chem. 1990, $29,2501$.

(32) Jaw, H. R.; Chang, T. H.; Zink, J. I. Inorg. Chem. 1987, 26, 4204. 